\title{
TIEMPO, ASPECTO Y MODO EN LOS CRIOLLOS INGLESES DEL ATLÁNTICO
}

\author{
Mario Portilla
}

\begin{abstract}
RESUMEN
Este estudio presenta una descripción sincrónica comparativa del sistema de tiempo, aspecto y modo de los criollos ingleses del Atlántico.
\end{abstract}

\begin{abstract}
This study is a synchronic comparative description of the tense, aspect and mood system corresponding to the English-based Atlantic Creoles.
\end{abstract}

\section{Introducción}

El presente estudio se ocupa de la descripción sincrónica comparativa de las funciones semánticas del sistema de tiempo, aspecto y modo (TAM) de las variedades criollas inglesas del Atlántico. Esta descripción se basa en datos tomados de las siguientes variedades: Gullah ${ }^{1}$ (Turner 1949), CI de Bahamas (Shilling 1975; Holm 1982), CI de Belice (Young 1973; Hellinger 1979; Escurre 1983 [Textos]), CI Nicaragua (Holm 1978; Holm 1983 [Textos]), CI de San Andrés (O’Flynn 1990; datos de trabajo de campo), CI de Costa Rica (Herzfeld 1978; Herzfeld 1983a [Textos]; Herzfeld 1983b; datos de trabajo de campo), CI Panamá (Herzfeld 1983a [Textos]; Herzfeld 1983b; datos de trabajo de campo), CI de Jamaica (Bailey 1966; Mufwene 1984), CI de Barbados (Roy 1986), CI de Tobago (Southers 1975), CI de Guyana (Mufwene 1984; Gibson 1986), sranan (Voorhoeve 1957; Seuren 1983), saramaca² (Byrne 1987), krio (Williams 1976; Jones 1990), wescos ${ }^{3}$ (Agheyisi 1971; Obilade 1981 [Textos]).

En primer lugar, se analiza el verbo simple con el fin de determinar los valores semánticos predominantes de este. En segundo lugar, se estudian las funciones semántica y pragmático-discursiva de los marcadores de tiempo tanto en la variedades basilectales como en las mesolectales. En tercer lugar, se describen los usos semánticos de los marcadores de las diferentes categorías aspectuales que se presentan en las variedades en estudio. Por último, se enumeran los diferentes auxiliares que expresan las distintas categorías modales presentes en las lenguas criollas comparadas. 


\section{El verbo simple: la forma no marcada}

La mayoría de los autores han señalado que, en las variedades comparadas, la interpretación temporo-aspectual asignada al verbo simple depende de la naturaleza semántica de este. Voorhoeve (1957) fue el primero en señalar la importancia de la distinción entre verbos dinámicos y verbos estativos para tal interpretación ${ }^{4}$.

Bickerton (1979: 24) define la interpretación semántica del verbo simple en los criollos atlánticos de la siguiente manera: “The stem form signifies past with nonstatives and nonpast with statives". Bickerton (cf. especialmente 1980 y 1981) sostiene, además, que esta función semántica del verbo simple es prototípica, e incluso quizá universal, de todas las lenguas criollas.

Desde una perspectiva pragmático-discursiva, Givón (1982 y 1984 [cap. 8]) señala que la interpretación semántica temporal ofrecida por Bickerton es parcial e incompleta, ya que no se ajusta a casos de verbos dinámicos en los que la interpretación puramente temporal de pasado es totalmente imposible. De este modo, Givón añade una caracterización pragmática de las funciones de los verbos simples: refieren eventos que ocurren en secuencia, que ofrecen la información principal y que constituyen el hilo fundamental de la narración.

\footnotetext{
The characterisation of the Ø-form as 'past' for action verbs is thus insufficient, though its characterisation as marking the 'in-sequence' portion of 'main clauses of 'backbone-narrative' is reasonably intact. Having said this one must concede that there are good reasons, grounded in the dynamics of narrative as a discourse style, why most of the backbone-foreground clauses may be easily interpreted as 'past': Narratives are largely told about events that occurred in the past. (Givón 1982:121)
}

La caracterización semántica del verbo simple ofrecida por Bickerton puede, además, ser completada con una perspectiva más orientada hacia el sentido aspectual inherente a los verbos dinámicos y estativos. Por una parte, prototípicamente, los verbos dinámicos expresan eventos que tienden a ser menos estables en el tiempo; es decir, que tienden a presentar una duración temporal más limitada, tales como golpear, aplaudir, caerse, etc. Los verbos dinámicos presentan, por así decirlo, inherentemente un aspecto más puntual.

Los verbos estativos, por otro lado, expresan estados que tienden a ser más estables en el tiempo, a tener una mayor duración temporal, tales como vivir, llamarse, tener, etc. Los verbos estativos presentan, pues, inherentemente un aspecto durativo o no puntual.

De esta forma, los eventos puntuales, cuyos límites en el tiempo están naturalmente más restringidos, tienen mayor probabilidad de corresponder a eventos pasados. Por el contrario, los estados no puntuales, cuyos límites en el tiempo tienden a ser naturalmente más amplios, ofrecen una mayor probabilidad de corresponder a estados presentes.

Desde el punto de vista de la naturalidad lingüística, se podría decir que la manifestación más natural de un verbo dinámico es expresar un evento puntual (en el pasado), mientras que la de un verbo estativo es expresar un estado durativo (que podría extenderse hasta el presente).

En las variedades criollas inglesas del Atlántico, que constituyen el presente objeto de estudio, el verbo simple presenta otras funciones semánticas, además de las prototípicas ya mencionadas. 
Funciones semánticas del verbo simple con verbos dinámicos:

1. Expresa un evento puntual (en el pasado).

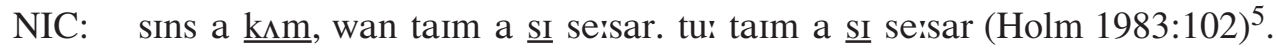
(Desde yo venir una vez yo ver Caesar. Dos vez yo ver Caesar.)

Desde que vine he visto a Caesar una vez. Dos veces he visto a Caesar.

El verbo simple con valor puntual ocurre en todas las variedades comparadas.

2. Expresa una acción habitual (en el presente o en el pasado).

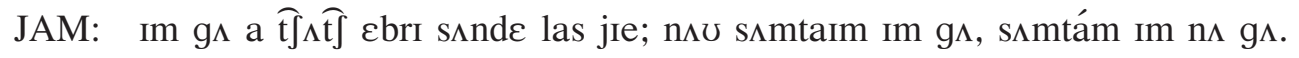
(Mufwene 1984: 206).

(Él ir a iglesia cada domingo último año; ahora a-veces él ir, a-veces él no ir.) Él iba a la iglesia todos los domingos el año pasado; ahora a veces va, a veces no va.

El valor habitual del verbo simple aparece en las siguientes variedades: CI de Belice, CI de Nicaragua, CI de San Andrés y Providencia, CI de Costa Rica, CI de Panamá, CI de Jamaica, CI de Barbados, CI de Tobago y krio. Y está ausente en las siguientes variedades: gullah, CI de Bahamas, CI de Guyana, sranan, saramaca y wescos.

Es importante señalar que existen diferencias entre las variedades en la frecuencia de uso del verbo simple para indicar predicaciones habituales. En el criollo de Costa Rica, por ejemplo, el verbo simple es el marcador prototípico de las expresiones habituales; en krio, por el contrario, el uso del verbo simple para expresar predicaciones habituales es más bien marginal, tratándose probablemente de una innovación reciente (Willams 1976: 227 y ss.)

Funciones semánticas del verbo simple con verbos estativos:

1. Expresa un estado en el presente:

COS: wá ju níem? (Datos de trabajo de campo)

(Qué tú llamarse.)

¿Cómo te llamas?

Este valor semántico aparece en todas las variedades comparadas.

2. Expresa un estado en el pasado:

KRI: a sik las wik (Jones 1990:846).

(Yo estar-enfermo última semana.)

Estuve enfermo la semana pasada. 
Las variedades que expresan estados en el pasado con el verbo simple son las siguientes: gullah, CI de Belice, CI de Nicaragua, CI de San Andrés y Providencia, CI de Costa Rica, CI de Panamá, CI de Jamaica, CI de Barbados, CI de Tobago, CI de Guyana, krio y wescos. Las variedades que no lo permiten son: sranan y saramaca.

Es importante señalar que el uso del verbo simple para expresar estados en el pasado es variable, a juzgar por el análisis de los textos disponibles así como, en general, por los comentarios de los autores en sus estudios sobre las variedades.

Por ejemplo, en textos del criollo de Belice ${ }^{6}$, de una muestra de 30 verbos estativos con sentido de pasado, 23 (77\%) presentan un marcador preverbal de tiempo y solo 7 (23\%) aparecen expresados con el verbo simple. En un texto del criollo de Costa Rica ${ }^{7}$, del mismo total de verbos estativos con sentido de pasado, 13 (43\%) presenta un marcador de tiempo, mientras que 17 (57\%) no lo presenta.

En ese mismo texto, el total de esos 17 verbos estativos sin marcador de tiempo aparece en un contexto en el que está explícita la referencia temporal al pasado.

COS: wat giem ju: jurstu plie wen ju smail? (Herzfeld 1983a: 135).

(Qué juego tú HAB-PAS jugar cuando tú ser-pequeño?).

¿Qué juego solías jugar cuando eras pequeño?

En el ejemplo anterior, el verbo estativo sma:l (ser-pequeño) hace referencia al pasado y aparece sin marcador alguno. El contexto, en este caso la forma verbal jurstu plie (HABPAS jugar), permite la correcta interpretación semántica del verbo estativo.

Por último, el verbo simple también permite expresar predicaciones con sentido temporal de futuro, tanto con verbos dinámicos como con verbos estativos.

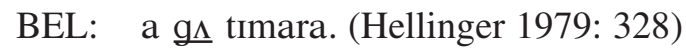

(Yo ir mañana.)

Yo voy a ir mañana.

COS: wí: av a kampáina tumára náit. (Datos de trabajo de campo)

(Nosotros tener una reunión mañana noche.)

Tendremos una reunión mañana por la noche.

El uso del verbo simple con sentido temporal de futuro es realmente excepcional. No aparece mencionado como un uso típico del verbo simple en ninguno de los estudios consultados sobre las variedades comparadas. Esto se debe, sin duda alguna, al hecho de que en estas lenguas el tiempo futuro es categorizado no como un tiempo propiamente dicho, sino más bien como un modo irrealis, para cuyo caso, como se verá más adelante, se utiliza un marcador modal determinado.

Sin embargo, es importante resaltar el hecho de que el verbo simple permita expresar eventos con sentido de futuro, ya que es un indicio de que, prototípicamente, el sentido básico que aporta el verbo simple no es temporal (la distinción entre presente/pasado), sino del aspecto no marcado que corresponde intrínsicamente a los verbos dinámicos y estativos (la distinción entre puntual/no puntual). 
En resumen, las funciones semánticas predominantes del verbo simple parecen estar más orientadas a expresar el sentido aspectual inherente de los verbos que a ofrecer un sentido temporal definido. En este sentido, el contexto juega un factor fundamental en la determinación de la interpretación temporal de los verbos simples.

\section{El tiempo}

El tiempo es expresado por medio de un auxiliar preverbal. Las variedades más basilectales presentan como marcador temporal una forma que la mayoría de los autores considera derivada del inglés $b e e n^{8}$. Esta forma aparece manifestada fonológicamente en las distintas variedades comparadas de la siguiente manera: bin (CI de Bahamas, CI de Barbados, CI de Tobago, CI de Guyana), bın (krio, wescos), bınə (gullah), bi (saramaca), In (CI de Tobago), ben (CI de Panamá, CI de Jamaica) ben (sranan), en (CI de Jamaica), min (CI de Tobago), mi (CI de Belice, CI de Nicaragua), men (CI de San Andrés, CI de Jamaica), me (CI de Belice), wen (CI de San Andrés, CI de Jamaica) ${ }^{9}$.

SAR: a bi méni di ótó. (Byrne 1987:45)

(Él TPO recordar la historia.)

Él recordó la historia.

Variedades que son consideradas más mesolectales presentan las siguientes formas como marcadores temporales: una forma derivada del verbo auxiliar inglés did, fonológicamente expresada como $d I d$ o $d I$.

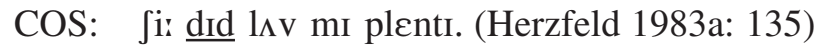

(Ella TPO amar me mucho.)

Ella me quería mucho.

Y la forma waz, con su variante $w \Lambda Z$, derivada del inglés was. Esta forma ocurre típicamente con verbos estativos, aunque en algunas variedades aparece también con verbos dinámicos (por ejemplo, en Bahamas).

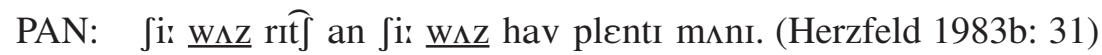

(Ella TPO ser-rica y ella TPO tener mucho dinero.)

Ella era rica y tenía mucho dinero.

Estas dos formas ${ }^{10}$ ocurren en gullah, CI de Bahamas, CI de Belice, CI de Nicaragua, CI de San Andrés, CI de Costa Rica, CI de Panamá, CI de Jamaica, CI de Barbados, CI de Tobago y CI de Guyana. Están ausentes en sranan, saramaca, krio ${ }^{11}$ y wescos. Las variedades en estudio pueden ser clasificadas tipológicamente en dos grupos de acuerdo con la función semántica del marcador de tiempo.

Para un grupo, el auxiliar temporal funciona como un marcador de tiempo anterior. Bickerton (1979: 309) define el tiempo anterior de la siguiente manera: [...] for statives [...] 
(may) indicate a simple past, but that for non statives, it must indicate a 'past before the past' something like (though by no means identical with) English pluperfect.

Desde un punto de vista tipológico, el tiempo anterior en estas variedades corresponde a lo que Comrie (1985:64 y ss.) ha definido como un tiempo absoluto-relativo; es decir, un tiempo que, por una parte, toma como punto de referencia el eje temporal absoluto (el acto de la enunciación, el presente), pero que, por otra parte, también toma como punto de referencia un eje temporal relativo (un evento mencionado en el contexto).

Es importante mencionar que tanto Bickerton (1975, 1979, 1981 inter alia) como Givón (1982 y 1984 [cap. 8]) sostienen el carácter prototípico y quizá universal de la presencia del tiempo anterior en las lenguas criollas, como consecuencia del proceso de criollización.

Las variedades que conforman este primer grupo son: sranan, saramaca y wescos. Además, pueden ser también consideradas las variedades llamadas más basilectales del CI de Belice, CI de Nicaragua, CI de San Andrés, CI de Costa Rica, CI de Panamá, CI de Jamaica, CI de Barbados, CI de Tobago y CI de Guyana.

Con verbos estativos:

SRA: $\quad$ ssde a ben wani go drape. (Echteld 1961: 60)

(Ayer él TAN querer ir allá.)

Ayer él quería ir allá.

Con verbos dinámicos:

SRA: mamanten dow ben ari gwe kba. (Seuren 1983: 239)

(Temprano-en-la-mañana rocío TAN halarse irse ya.)

Temprano en la mañana el rocío ya se había secado.

Con los verbos estativos, la presencia del marcador de anterioridad tiene carácter obligatorio en sranan y saramaca.

En CI de Belice, CI de Nicaragua, CI de San Andrés, CI de Costa Rica, CI de Panamá, CI de Jamaica, CI de Barbados, CI de Tobago, CI de Guyana y wescos el marcador de anterioridad, con verbos estativos, no es obligatorio.

En algunos de los textos de los criollos de América Central (Holm (ed.) 1983), el uso del marcador parece estar determinado pragmáticamente. Así, por ejemplo, en un texto del CI de Panamá (Herzfeld 1983a), de 17 verbos estativos que presentan el marcador temporal (de un total de 22), 12 ocurren en un contexto donde ha ocurrido un cambio de tópico. El cambio de tópico parece favorecer, pues, la presencia del marcador temporal.

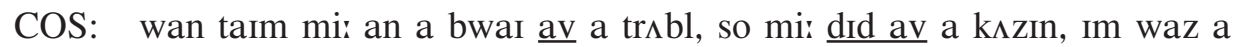
baksa, kım fram panama, an Im lark echar carbón pan jui, nuo? (Herzfeld 1983a:137)

(Una vez yo y un muchacho tener un problema, así yo TAN tener un primo, él ANT un boxeador, venir de Panamá, y él gustar echar carbón sobre tú, saber?) Una vez un muchacho y yo tuvimos un problema, pues yo tenía un primo, él era boxeador, venía de Panamá, y a él le gustaba provocarte, sabes? 
En el ejemplo anterior, el primer verbo estativo ( $\underline{a v}$ a tra $b I$ ) aparece sin marcador tem-

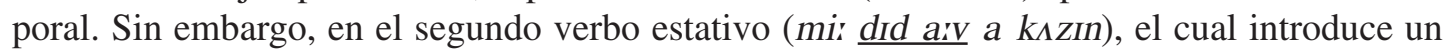
nuevo tópico, se presenta el marcador preverbal de tiempo.

El cambio de tiempo en la narración parece favorecer también el uso del marcador temporal. En el texto del criollo de Panamá, de los 17 verbos con marcador temporal, 10 ocurren en un contexto donde ha ocurrido un cambio de tiempo en la narración (cambio de presente a pasado).

COS: - wat ju rimemba bsut skuil die?

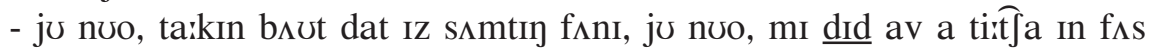
gried... (Herzfeld 1983a:136)

(- ¿Qué tú recordar sobre escuela días?

- Tú saber, hablar sobre eso ser algo divertido, tú saber, yo TAN tener una maestra en primer grado...)

- ¿Qué recuerdas acerca de los días de escuela?

- Sabes, hablar acerca de eso es algo divertido, sabes, yo tenía una maestra en primer grado...

Por último, el uso del marcador temporal parece tener también a veces una función de énfasis.

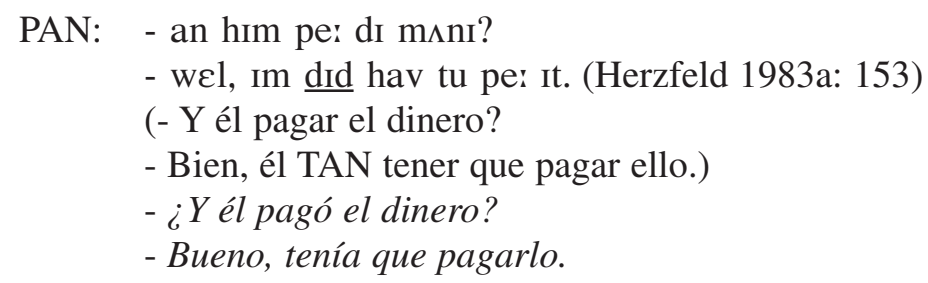

Para otro grupo, el auxiliar temporal funciona como un marcador de tiempo pasado. En este caso, el tiempo pasado es definido como un tiempo absoluto (Comrie 1982: 36 y ss.). Es decir, como un tiempo que toma como punto de referencia solamente el eje temporal absoluto. El marcador de tiempo pasado indica simplemente que el evento ha ocurrido en un momento anterior al acto de la enunciación.

Se debe señalar que en estas variedades el uso del marcador de tiempo pasado tiene carácter optativo.

Este grupo está conformado por las siguientes variedades: gullah, CI de Bahamas, krio y por variedades consideradas quizá más mesolectales del CI de Belice, CI de Nicaragua, CI de San Andrés, CI de Costa Rica, CI de Panamá, CI de Jamaica, CI de Barbados, CI de Tobago y CI de Guyana.

En síntesis, se puede señalar que, con verbos dinámicos, la función semántica fundamental del tiempo es la de hacer referencia a un evento ocurrido fuera de la secuencia temporal cronológica en que ocurren los hechos narrados. Con verbos estativos, por otra parte, el señalamiento del tiempo está condicionado por factores contextuales, especialmente por el cambio de tópico y por el cambio de tiempo en la narración. Por otro lado, no existe ninguna 
diferencia semántica entre los distintos marcadores de tiempo, aunque sí la hay en la definición semántica del tiempo mismo en las distintas variedades comparadas, incluyendo presumiblemente diferencias entre los basilectos y los mesolectos. Esto permite suponer que, en algunas variedades, está ocurriendo un cambio en proceso por el cual la categoría de tiempo anterior es interpretada como un simple tiempo pasado.

\section{El aspecto}

\subsection{El aspecto progresivo}

El aspecto progresivo indica que la situación referida por el verbo se encuentra en progreso y que, por lo tanto, presenta continuidad durante un limitado período de tiempo. Este aspecto es expresado por medio de un auxiliar preverbal en variedades que pueden ser consideradas más basilectales. Los marcadores preverbales que presentan las variedades comparadas son los siguientes:

1. La forma da, con su variantes do y a.

TOB: di ol man a ded. (Southers 1975: 79)

(El viejo hombre PRO morir.)

El viejo está muriendo.

Esta forma es homófona con la cópula ecuativo/identificativa y con el marcador de topicalizador de la mayoría de las variedades más basilectales de criollo (Cf. Holm 1980).

No existe consenso en cuanto a la etimología de esta forma. Arens (1986) la deriva del pronombre inglés that. Mufwene (1984) propone que proviene de la proposición locativa inglesa at. Holm (1982) considera la posibilidad de influencia de un marcador de progresivo [ ə- ] de uso arcaico y dialectal en inglés.

Las variedades en las que aparece esta forma son: CI de Bahamas, CI de Nicaragua, CI de Costa Rica, CI de Jamaica, CI de Barbados, CI de Tobago y CI de Guyana.

2. La forma $\mathrm{d} \varepsilon$, con sus variantes $d e, \varepsilon \mathrm{y}$ di.

SAN: aI, a kja:n wa:k, mi belı de hıt mi. (Datos de trabajo de campo) (Ay, yo MOD-HAB/NEG caminar, mi estómago PRO doler me.)

Ay, no puedo caminar, me está doliendo el estómago.

Esta forma es homófona con la cópula locativo/existencial de todas las variedades comparadas.

La mayoría de los autores (Echteld 1961; Arends 1986; Mufwene 1984 inter alia) concuerdan en derivar esta forma del adverbio locativo inglés there. Holm (1982) señala también una posible convergencia con el marcador de progresivo en diversas lenguas de África Occidental, por ejemplo re en $t w i$, de en ewe, di en wolof. 
Las variedades que presentan estas formas son: CI de Belice, CI de San Andrés, CI de Panamá, sranan, krio y wescos ${ }^{12}$.

3. La forma tá.

SAR: a tá woóko. (Byrne 1987: 47)

(Él PRO trabajar.)

Él está trabajando.

Existe absoluto consenso en derivar esta forma del portugués estar. Esta forma ocurre solamente en saramaca. sufijo - In.

4. El aspecto progresivo también puede ser expresado posverbalmente por medio del

COS: ái, jú: đ̧̧̋́rkin mi, a sík. (Datos de trabajo de campo) (Ay, tú llevar-a-tirones-PRO me, yo estar-enfermo.)

Ay, me estás llevando a tirones, yo estoy enfermo.

Esta forma proviene, sin duda alguna, del morfema -ing, marcador del llamado participio presente en inglés. Este morfema ha sido considerado un marcador típico de las variedades mesolectales. Este aparece en las siguientes variedades: gullah, CI de Bahamas, CI de Belice, CI de Nicaragua, CI de San Andrés, CI de Costa Rica, CI de Panamá, CI de Jamaica, CI de Barbados, CI de Tobago y CI de Guyana. Y está ausente en sranan, saramaca, krio y wescos.

En estas variedades, un mismo verbo puede presentar optativamente ambos marcadores de aspecto progresivo a la vez.

GUL: $\quad$ sista, sopm də kımㅍ․ (Turner 1949: 263)

(Hermana, algo PRO venir-PRO)

Hermana, algo está viniendo.

En todas las variedades comparadas, la combinación del marcador de aspecto progresivo y el marcador de tiempo (ya sea como anterior o pasado) con verbos dinámicos denota característicamente una situación que tiene como punto de referencia el eje temporal absoluto. Esto se debe seguramente al hecho de que una de las funciones semánticas del progresivo es darle un sentido aspectual durativo a los verbos dinámicos. Por lo tanto, es lógico que un verbo dinámico en progresivo se comporte como un verbo estativo, intrínsecamente durativo.

BEL: a $\underline{m \varepsilon} \underline{\mathrm{d} \varepsilon}$ wa:k ina bvj dis mainin. (Escurre 1983: 46)

(Yo TPO PRO caminar dentro bosque esta mañana.)

Yo estuve caminando en el bosque esta mañana. 
Además, en las variedades cuyo uso del marcador temporal es optativo, es posible también expresar situaciones progresivas con sentido de pasado sin el marcador preverbal de tiempo.

TOB: wen bre tekuma go we hi a kıt pis a bre larjan an hi a kuk.

(Southers 1975: 237)

(Cuando hermano Tecuma ir lejos él PRO cortar pedazo de hemano léon y él PRO cocinar.)

Cuando el hermano Tecuma se fue, él [Anansi] estuvo cortando un pedazo del hermano León y cocinándolo.

Además, el aspecto progresivo puede cubrir en algunos casos también predicaciones con sentido aspectual habitual o iterativo.

KRI: modupe de gó krúton ród mákit évri mónde. (Williams 1976: 224)

(Modupe PRO ir Krootown Road mercado cada lunes.)

Modupe está yendo al mercado de Krootown Road todos los lunes.

Esta función semántica del progresivo ha sido registrada en todas las variedades comparadas, con excepción de Panamá13.

Por último, aunque normalmente el aspecto progresivo aparece normalmente solo con verbos dinámicos, a veces el marcador de progresivo puede ocurrir también con algunos verbos estativos. En este caso, el verbo no expresa ya una situación estativa, sino más bien una situación progresiva con valor aspectual habitual o incoativo.

Esta función semántica del marcador de progresivo aparece en las siguientes variedades: CI de San Andrés, CI de Guyana, sranan y saramaca.

SAR: a tá sábi di tóỹgo. (Byrne 1987: 46)

(Él PRO saber la lengua.)

Él está sabiendo la lengua. (i.e. Él está empezando a saber la lengua.)

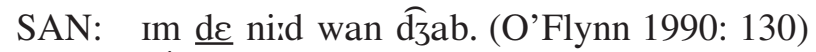

(Él PRO necesitar un trabajo.)

Él está necesitando un trabajo.

\subsection{El aspecto durativo}

El aspecto durativo indica que la situación referida por el verbo ocurre de manera constante e ininterrumpida durante un cierto período.

El marcador de aspecto durativo es la forma $d \varepsilon$ para las variedades más basilectales. Como se ha dicho, está forma es homófona con la cópula locativo/ existencial y se deriva problamente del inglés there. Esta aparece en CI de Bahamas, CI de Nicaragua, CI de Jamaica, CI de Tobago y CI de Guyana. 
TOB: $\quad$ mi in $\underline{\mathrm{d} \varepsilon} \mathrm{r} \wedge \mathrm{n}$. (Southers 1975: 209)

(Yo TPO DUR correr.)

Yo estuve corriendo.

Este marcador coincide con el marcador de aspecto progresivo en algunas de las variedades comparadas. Según Southers (1975), la diferencia fundamental del aspecto durativo con respecto al progresivo reside en que la función semántica básica del primero es señalar la cohesión y el grado de estabilidad temporal del evento, mientras que la función semántica del segundo es indicar simplemente imperfectividad.

Por esta razón, no es extraño que el marcador de progresivo pueda cubrir también predicaciones con sentido habitual, lo cual no es posible conseguir con el marcador de aspecto durativo.

Como se ha mencionado, el marcador de progresivo también puede ser compatible, algunas veces, con verbos estativos. El marcador de aspecto durativo, sin embargo, solamente es compatible con verbos dinámicos, ya que rechaza toda posible interpretación habitual o iterativa, es decir, la recurrencia de una situación a intervalos.

Por otra parte, el marcador de durativo puede aparecer en un mismo verbo en combinación con los marcadores de aspecto progresivo y de aspecto habitual.

GUY: Ji: $\underline{a} \underline{\mathrm{d} \varepsilon} \underline{\mathrm{a}} \sin$. (Gibson 1986: 572)

(Ella HAB DUR PRO cantar.)

Ella suele estar cantando.

El ejemplo anterior muestra claramente que el aspecto durativo existe independientemente del aspecto progresivo (y del aspecto habitual) en las variedades mencionadas, a pesar de que las glosas no permitan reflejar claramente su diferencia semántica.

\subsection{El aspecto habitual}

El aspecto habitual describe una situación que es característica de un período extendido de tiempo (Comrie 1976: 26 y ss.). La expresión de la habitualidad cubre un espectro que va desde una situación genérica o atemporal (habitual-genérico) hasta una situación repetitiva temporalmente limitada (habitual-iterativo).

Las variedades comparadas expresan las predicaciones habituales de las siguientes formas posibles:

Por medio de un marcador que es exclusivo del aspecto habitual. En la mayoría de las variedades, este es la forma $d \Lambda z$, con sus variantes $\Lambda z, z$, entre otras, la cual se deriva del verbo auxiliar inglés does (Rickford 1986: 269). Esta forma aparece en las siguientes variedades: gullah, CI de Bahamas, CI de Belice ${ }^{14}$, CI de Nicaragua, CI de San Andrés, CI de Panamá, CI de Barbados, CI de Tobago y CI de Guyana.

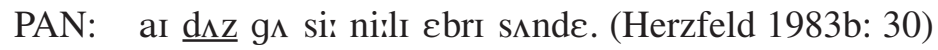

(Yo HAB ir mar casi cada domingo.)

Yo suelo ir al mar casi todos los domingos. 
En krio ${ }^{15}$, el marcador exclusivo de aspecto habitual es la forma kin, la cual se derivaría del verbo modal inglés can (Hancock 1969: 36-7).

KRI: mi mamá kin gó mákit sátide. (Williams 1976: 223)

(Mi madre HAB ir mercado sábado.)

Mi madre suele ir al mercado los sábados.

En otras variedades, las predicaciones habituales son expresadas por medio del verbo simple: CI de Belice, CI de Nicaragua, CI de San Andrés, CI de Costa Rica, CI de Panamá, CI de Jamaica, CI de Barbados, CI de Tobago y krio.

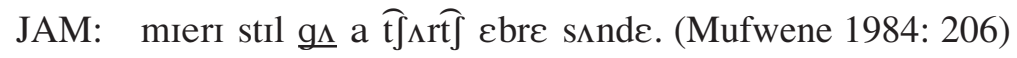

(Mary todavía ir a iglesia cada domingo.)

Mary todavía va a la iglesia todos los domingos.

La frecuencia del uso del verbo simple para expresar predicaciones habituales es distinta según las variedades. En CI de Belice, CI de Costa Rica y CI de Jamaica, esta es la forma típica de expresar el aspecto habitual. En krio, por el contrario, este uso del verbo simple es más bien ocasional. Otras variedades ocupan un lugar intermedio entre estos dos extremos.

Por último, como se ha dicho, en todas las variedades comparadas, el marcador de progresivo también permite expresar predicaciones con sentido habitual.

Sin embargo, en algunas variedades, este uso es ocasional y de ningún modo típico. Esto es válido, por lo menos, para el CI de Belice, CI de Nicaragua, CI de San Andrés, CI de Costa Rica, CI de Panamá y CI de Jamaica. En otras variedades, el marcador de progresivo es el único medio que existe para expresar habitualidad: sranan, saramaca y wescos.

WES: tif de tek wi moni. (Agheyisi 1971: 135)

(Ladrón PRO/HAB tomar nuestro dinero.)

Un ladrón está tomando (o toma) nuestro dinero.

En la mayoría de las variedades existe un marcador de aspecto habitual exclusivo para el pasado. Este es la forma juista, con sus variantes juisa y juistu, la cual se deriva seguramente del auxiliar de aspecto habitual pasado inglés used to. Esta forma existe en las siguientes variedades: Gullah, CI de Bahamas, CI de Belice, CI de Nicaragua, CI de San Andrés, CI de Costa Rica, CI de Panamá, CI de Jamaica, CI de Barbados, CI de Tobago y CI de Guyana. Y está ausente en sranan, saramaca, krio y wescos.

COS: $\quad$ wat grem ju: juistu ple wen ju: smail? (Herzfeld 1983a: 135)

(Qué juego tú HAB-PAS jugar cuando tú ser-pequeño?)

¿Qué juego te gustaba jugar cuando eras pequeño?

En algunas de las variedades que presentan este marcador exclusivo de pasado habitual es posible expresar también predicaciones habituales en el pasado sin este marcador. Esto ocurre, por lo menos, en las variedades consideradas más basilectales de CI de Bahamas, CI de Belice y CI de Guyana. 
BEL: wi: $\underline{\mathrm{d} \Lambda \mathrm{z}}$ liv da malanti. (Young 1973: 259)

(Nosotros HAB vivir en Malanti.)

Nosotros vivíamos en Malanti.

El marcador $d_{\Lambda} z$ nunca puede aparecer combinado con el marcador de tiempo. Sin embargo, en algunas variedades, el marcador jussta, optativamente, sí puede hacerlo: Nicaragua, Costa Rica y Panamá.

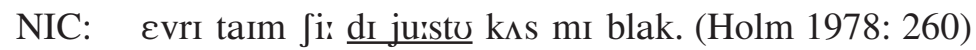

(Cada vez ella TPO HAB-PAS insultar me negro.)

Cada vez ella me insultaba diciéndome negro.

\subsection{El aspecto completivo}

El aspecto completivo indica que la situación referida por el verbo está concluida completamente. Otros términos que han sido usados para designar este aspecto son: terminativo (Mufwene 1984), conclusivo (Dahl 1985) y perfectivo (Jones 1971).

Tal como ha sido definido por Comrie (1976), el término perfectivo en este caso parece ser inadecuado para designar este aspecto. El aspecto perfectivo designa una situación concluida que es presentada como un todo, sin referirse a ninguna de las partes componentes de la situación (inicio-desarrollo-fin), mientras que el aspecto completivo designa particularmente el fin de la situación concluida, es decir su compleción (Holm 1988: 162).

En la mayoría de las variedades comparadas, el marcador de aspecto completivo es la forma $d \Lambda n$, la cual se derivaría del inglés done. Este marcador es homófono con el verbo correspondiente al significado de acabarse, terminarse. Aparece en gullah, CI de Bahamas, CI de Belice, CI de Nicaragua, CI de San Andrés, CI de Costa Rica, CI de Panamá, CI de Jamaica, CI de Barbados, CI de Tobago, CI de Guyana, krio y wescos. Y está ausente en sranan y saramaca.

NIC: $\quad$ aI $\underline{\mathrm{d} \Lambda \mathrm{n}}$ giv Im maI darrekJan. (Holm 1978: 261)

(Yo COM dar él mi dirección.)

Yo ya le di mi dirección.

En todas las variedades, este marcador ocurre en posición preverbal. En CI de Jamaica, CI de Guyana y krio, además, puede ocurrir en posición posverbal.

KRI: wen a it don, a (bin) go toy. (Jones 1990: 847)

(Cuando yo comer COM, yo (PAS) ir pueblo.)

Cuando acabé de comer, fui al pueblo.

Según Mufwene (1984), esta forma en posición posverbal tiene más bien carácter de verbo pleno y constituye simplemente una construcción verbal serial. 
JAM: mi ta:k $\underline{\mathrm{d} \Lambda \mathrm{n}}$. (Mufwene 1984: 208)

(Yo hablar acabar.)

Yo terminé de hablar.

El aspecto completivo es expresado en sranan y saramaca por la forma kaba, con su variante $k b a$. Esta se deriva con seguridad del verbo portugués acabar.

SRA: mi kba nan. (Seuren 1983: 248)

(Yo COM comer.)

Yo acabé de comer.

En ambas variedades, esta forma también puede ocurrir en posición posverbal. Según Seuren, en sranan, esta forma, en posición posverbal, tiene más bien carácter adverbial, con el significado aproximado del adverbio ya.

SRA: mi nam kba. (Seuren 1983: 248)

(Yo comer ya [?])

Yo ya comí.

Por último, el marcador de aspecto completivo puede aparecer combinado con el marcador de aspecto progresivo y con el marcador de tiempo, pero solamente cuando ocurre en posición preverbal.

JAM: $\widehat{d} \overline{3} a: \widehat{d} \bar{b} \underline{b} \underline{n} \underline{a} \underline{\mathrm{d} \Lambda \mathrm{n}}$ rait Im asainment. (Mufwene 1984: 209)

(George TPO PRO COM escribir su tarea.)

George estaba acabando de escribir su tarea.

En resumen, en términos generales se puede afirmar que, en las variedades criollas comparadas, el énfasis que se da en la marcación de las categorías aspectuales es mucho más sobresaliente que el que se le da a la categoría de tiempo. La mayoría de las variedades presenta cuatro categorías aspectuales señaladas gramaticalmente por marcadores independientes: progresivo, durativo, habitual y completivo. Las categorías de progresivo y de completivo parecen ser las más generales, ya que en algunas variedades las categorías de durativo y de habitual tienden a confundirse con la de progresivo.

\section{La modalidad ${ }^{16}$}

\subsection{La modalidad factual}

La modalidad factual indica que la situación referida por el verbo es considerada un hecho real. Esta modalidad verbal carece de marcador auxiliar modal alguno; es decir, corresponde a la forma no marcada del verbo. La modalidad factual aparece expresada de esta forma en todas las variedades comparadas. 
WES: meri get plenti moni. (Agheyisi 1971: 133)

(Mary tener mucho dinero.)

Mary tiene mucho dinero.

Además, la modalidad factual incluye predicaciones con sentido imperativo. El imperativo aparece, pues, expresado sin marcador alguno en todas las variedades comparadas.

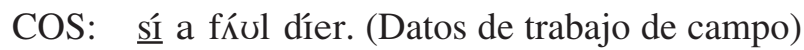

(Ver una gallina ahí.)

Ve una gallina ahí.

\subsection{La modalidad irrealis}

En el modo irrealis, la situación expresada por el verbo es un hecho que no tiene contingencia real, ya sea porque no ha ocurrido ${ }^{17}$, ya sea porque no se tiene certeza de su ocurrencia. El modo irrealis puede ser expresado por medio de los siguientes marcadores:

La forma sa, la cual, según Echteld (1961:87) y otros, podría derivarse del auxiliar inglés shall o del auxiliar holandés zal. Esta forma aparece en sranan y CI de Guyana. No ha sido registrada en las demás variedades comparadas.

SRA: m sa tak nay a bakra. (Voorhoeve 1957: 379)

(Yo IRR hablar con el hombre-blanco.)

Yo hablaré con el hombre blanco.

La forma wIl, con su variante $W I$, se deriva seguramente del auxiliar inglés will. Esta forma aparece en variedades consideradas más mesolectales de CI de Belice, CI de Nicaragua, CI de San Andrés, CI de Costa Rica, CI de Panamá y CI de Jamaica ${ }^{18 .}$

NIC: diz baistad wi mIlk ju. (Holm 1978: 262)

(Estos bastardos IRR chupar te.)

Estos bastardos te van a desplumar.

La forma PRO + gh, con sus variantes $g a, g u, o$, se deriva del verbo inglés go. Esta forma, con o sin el marcador de progresivo, aparece en las variedades más basilectales de CI de Bahamas, CI de Nicaragua, CI de Jamaica, CI de Barbados, CI de Tobago, CI de Guyana, sranan y krio. Además, la forma sin el marcador de progresivo, ocurre en saramaca y wescos.

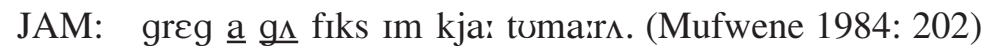

(Greg PRO/IRR arreglar su auto mañana.)

Greg va a arreglar su auto mañana.

En variedades presumiblemente más mesolectales, aparece la forma gwain, con sus variantes gwen, wa:n, gain, g^n. Esta forma se deriva del participio presente inglés going, que es la forma progresiva del verbo inglés go. Esta forma aparece en gullah, CI de Belice, CI de 
Nicaragua, CI de San Andrés, CI de Costa Rica, CI de Panamá, CI de Jamaica, CI de Barbados y CI de Guyana.

\author{
GUL: aI gwain kıs əm, ju no. (Turner 1949: 261) \\ (Yo IRR/PRO maldecir a él, tú saber.) \\ Lo voy a maldecir, sabes.
}

Los marcadores $s a$ y PRO $+g \Lambda$ (con su variante $g$ waIn) pueden ocurrir precedidos por el marcador de tiempo. En este caso, el verbo refiere una situación condicional, es decir, una situación que ocurre en un tiempo posterior a un punto de referencia que se ubica en un tiempo anterior al momento de la enunciación. El verbo indica, pues, un futuro en el pasado ${ }^{19}$. La situación no tiene una contingencia factual real en relación con el punto de referencia ubicado en el pasado; por lo tanto, es una situación hipotética.

TOB: đ̧̧an se I $\underline{\text { min }} \underline{\text { a go }}$ ren? (Southers 1975: 209)

(John decir ello TPO PRO IRR llover?)

¿John dijo que iba a llover (que llovería)?

El marcador wIl presenta el alomorfo supletivo wod para referir esta situación condicional, el cual se derivaría del inglés would. Esta forma aparece en variedades presumiblemente más mesolectales de gullah, CI de Bahamas, CI de Belice, CI de Nicaragua, CI de San Andrés, CI de Costa Rica, CI de Panamá, CI de Jamaica, CI de Tobago y CI de Guyana.

TOB: $\quad \int \mathrm{i}$ wudn tel mi dat. (Southers 1975: 70)

(Ella CON/NEG decir me eso.)

Ella no me diría eso.

Además, en estas variedades existe otro auxiliar que expresa una situación condicional, la forma wuda, la cual probablemente se deriva del inglés would have (cf. Hellinger 1979: 321).

Este auxiliar expresa también una situación que ocurre en un momento posterior a otro evento sucedido en el pasado, pero además indica que el evento es anterior a un tercer evento que también ocurre en el pasado. Esta forma puede ser denominada un condicional anterior o perfecto.

COS: If a kudin swim, a wuda druun. (Herzfeld 1978: 298)

(Si yo MOD-HAB/TAN/NEG nadar, yo CON/TAN ahogarse.)

Si yo no hubiera sabido nadar, me habría ahogado.

Por último, es importante señalar que, por lo menos ocasionalmente, el auxiliar del modo irrealis WIl también puede expresar una situación condicional (un futuro en el pasado). Esto se explica por el hecho de que en algunas de estas variedades criollas la designación formal del tiempo es opcional. 
PAN: - fi $\Lambda \mho \operatorname{m} \Lambda \mathrm{t} \int$ de: ju wuda put Im ina prizin?

- wan dala fa wan de:.

- an wat Im wil it? banana an jam? (Herzfeld 1983a: 153)

(- Por cuántos días tú CON/TAN poner él en prisión?

- Un dólar por un día.

- Y qué él IRR comer? ¿Bananos y ñame?)

- ¿Cuántos días lo habrías puesto en prisión?

- Un dólar por día.

- ¿Y qué iba a comer (comería)? ¿Bananos y ñame?

\subsection{La modalidad epistémica inferencial}

El auxiliar modal expresa una conjetura o suposición acerca de un hecho. Esta modalidad es expresada por medio de la forma $m \Lambda s$, con sus variantes mos y musu, derivada del auxiliar modal inglés must, o por la forma musi, con su variante music, derivada probablemente del inglés must + be (Young 1973: 265).

Estas formas aparecen en CI de Bahamas, CI de Belice, CI de San Andrés, CI de Costa Rica, CI de Jamaica, CI de Tobago, CI de Guyana, sranan y krio.

SRA: a musu tru. (Seuren 1983: 229)

(Ello MOD-INF ser-verdad.)

Eso debe de ser cierto.

Algunas variedades presentan también una forma de auxiliar de modalidad inferencial exclusiva para el tiempo anterior/pasado, mı sa, derivada probablemente del inglés must + have. Esta forma ocurre en todas las variedades mencionadas anteriormente, excepto en sranan y en krio.

SAN: Im mısa get mını. (O’ Flynn 1990: 156)

(Él MOD-INF/TAN conseguir dinero.)

Él debióldebe haber conseguido dinero.

Por último, la modalidad inferencial puede ser expresada por las formas matt y maIta, derivadas del inglés might (+ have). Estas formas aparecen en CI de Bahamas, CI de Belice, CI de San Andrés, CI de Costa Rica, CI de Tobago y CI de Guyana.

TOB: $\quad$ mi maIt si Ji tude. (Southers 1975: 70)

(Yo MOD-INF ver ella hoy.)

Posiblemente la vea hoy.

\subsection{La modalidad deóntica de deber}

El auxiliar expresa la idea de deber moral o necesidad. Esta modalidad es expresada también por medio del auxiliar mıs, con sus variantes mos y musu. Así ocurre en todas las variedades en las que aparece este auxiliar modal. 
KRI: a mos go nau no fo mis di tren. (Jones 1990: 862) (Yo MOD-DEB ir ahora para no perder el tren.) Me debo ir ahora para no perder el tren.

Además, la modalidad deóntica de deber es expresada por el auxiliar fI, con sus variantes fa, fu, fo, fo. Esta forma es homófona con un completizador de infinitivos y con la preposición con sentido benefactivo.

Edwards (1974) y Washabaugh (1975) proponen que estas formas pueden derivarse del Twi fi, del Ewe fe o del Yoruba fun. Byrne (1984) sostiene, sin embargo, que estas se derivan de la preposición inglesa for. Este modal ha sido registrado en gullah, CI de San Andrés, CI de Nicaragua, CI de Jamaica, CI de Guyana, sranan, saramaca, krio y wescos.

JAM: ju ben fi si mi jeside, bıt ju neba kım. (Mufwene 1984: 213)

(Tú TPO MOD-DEB ver me ayer, pero tú no venir.)

Debías haberme visto ayer, pero no viniste.

En algunas variedades, existe además una forma alternativa que es exclusiva del tiempo anterior/pasado de esta modalidad de deber deóntico, Juda, la cual se deriva probablemente del inglés should + have. Esta forma expresa, además, la idea de una situación irreal. Aparece en CI de Bahamas, CI de Belice, CI de San Andrés, CI de Costa Rica, CI de Jamaica, CI de Barbados, CI de Tobago y CI de Guyana.

SAN: Im 〔uda kom If ju tel Im. (O’Flynn 1990: 165)

(Él MOD-DEB/TAN venir si tú decir él.)

Él debería venir si tú le dijeras.

\subsection{La modalidad deóntica de obligación}

El auxiliar expresa la idea de obligación ineludible. Esta modalidad puede ocasionalmente ser expresada también por los auxiliares de deber deóntico mıs y fI.

BEL: I $\underline{\text { m } \Lambda \text { s }}$ du it $\varepsilon v r I$ der. (Young 1973: 267)

(Él MOD-DEB hacer ello cada día.)

Él tiene que hacerlo todos los días.

Sin embargo, esta modalidad es expresada más típicamente por las formas hafı, con sus variantes hafa y hafu, derivada probablemente de la forma have + for; haftu, derivada de have + to; gatfu, con sus variantes getfu, derivada de got $($ get $)+$ for; y gatstu, derivada de gat + to.

Estas formas han sido registradas en CI de Bahamas, CI de Belice, CI de San Andrés, CI de Costa Rica, CI de Jamaica, CI de Tobago, CI de Guyana y krio.

TOB: mi hafa lef. (Southers 1975: 80)

(Yo MOD-OBL irse.)

Tengo que irme. 


\subsection{La modalidad deóntica de habilidad}

El auxiliar expresa la idea de la posibilidad de realizar un evento. En la mayoría de las variedades, esta modalidad es expresada por la forma kjan, con sus variantes kan y kin, derivadas del auxiliar modal inglés can. Este aparece en CI de Belice, CI de Nicaragua, CI de San Andrés, CI de Costa Rica, CI de Panamá, CI de Jamaica, CI de Tobago, CI de Guyana, sranan y krio.

PAN: ju kjan memba wat eir ju pupa ded? (Herzfeld 1983a: 152)

(Tú MOD-HAB recordar qué año tu papá morir?)

¿Puedes recordar en qué año murió tu papá?

En sranan, esta modalidad es expresada por medio del auxiliar man, el cual se deriva con seguridad del auxiliar modal holandés man.

SRA: a man kon. (Echteld 1961: 79)

(Él MOD-HAB venir.)

Él puede venir.

En wescos, esta modalidad es expresada por la forma auxiliar fit, derivada quizás del verbo inglés fit.

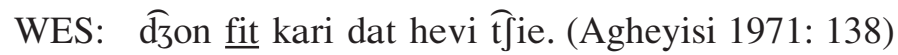

(John MOD-HAB cargar esa pesada silla.)

John puede cargar esa silla pesada.

En CI de Bahamas, CI de Nicaragua y CI de Barbados, esta modalidad se expresa por medio de la forma $k v d$, derivada del auxiliar modal inglés could.

NIC: hi d $\quad$ z trai hiz bes to du: wa hi kvd wid dem. (Holm 1978: 263)

(Él HAB tratar su mejor de hacer que MOD-HAB con ellos.)

Él trata de hacer lo mejor que puede con ellos.

En algunas variedades existe, además, una forma auxiliar exclusiva de tiempo anterior/pasado de esta modalidad, kuda, derivada probablemente del inglés could + have. Esta forma expresa también un sentido de irrealidad del evento. Ha sido registrada en CI de Bahamas, CI de Belice, CI de Nicaragua, CI de San Andrés, CI de Costa Rica, CI de Jamaica, CI de Barbados, CI de Tobago y CI de Guyana.

SAN: Im kuda plie gud. (O’Flynn 1990: 165)

(Él MOD-HAB/TAN jugar bien.)

Él pudo/podía/podría jugar bien. 
Para finalizar, se podría decir que tal vez el aspecto más sobresaliente en la descripción de la modalidad en las variedades comparadas está en el hecho de que en la mayoría de los casos existen dos o tres auxiliares diferentes para expresar una misma categoría modal. Por ejemplo, la modalidad irrealis se expresa a la vez por medio de los auxiliares PRO $+g \Lambda$ y wII y la modalidad deóntica de obligación es expresada por los auxiliares mıs, fi, haftu y hafi. Esto es un indicio de que, probablemente, en un estado primitivo de estas variedades, la modalidad no fuera una categoría tan estructurada como las categorías de tiempo y aspecto. Por otra parte, sin embargo, es notable que la mayoría de las variedades comparadas presenten las mismas categorías modales: modalidad irrealis, modalidad epistémica inferencial, modalidad deóntica de deber, modalidad deóntica de obligación y modalidad deóntica de habilidad.

\section{Conclusiones}

De acuerdo con los datos sincrónicos, solamente las variedades de Surinam están en concordancia con la descripción semántica del verbo simple ofrecida por Bickerton (1979); a saber, expresar pasado con verbos dinámicos y presente con verbos estativos. En todas las demás variedades, la forma simple del verbo con verbos estativos puede tener significado temporal tanto de presente como de pasado.

El análisis de textos de algunos criollos de América Central muestra que el uso del marcador de tiempo con verbos estativos está determinado por factores pragmático-discursivos. Por otra parte, el marcador de tiempo con verbos dinámicos siempre tiene valor semántico de pluscuamperfecto; es decir que indica un evento sucedido en un momento anterior a otro evento del contexto.

Por otro lado, el aspecto parece jugar en estas variedades un papel más preponderante que el tiempo. Todas las variedades presentan un aspecto progresivo y un aspecto completivo. Además, la mayoría ofrece también un aspecto habitual y un aspecto durativo. El aspecto progresivo puede servir para expresar también predicaciones habituales, inclusive en aquellas variedades que presentan marcadores independientes para ambos aspectos.

Por último, han sido encontradas cinco clases de modalidad no factual, las cuales son comunes a la mayoría de las variedades comparadas.

\section{Abreviaturas}

$\begin{array}{ll}\text { CI } & \text { Criollo inglés } \\ \text { COM } & \text { Aspecto completivo } \\ \text { CON } & \text { Modo condicional } \\ \text { DUR } & \text { Aspecto durativo } \\ \text { HAB } & \text { Aspecto habitual } \\ \text { HAB-PAS } & \text { Aspecto habitual pasado } \\ \text { IRR } & \text { Modo irrealis } \\ \text { MOD } & \text { Modal } \\ \text { MOD-DEB } & \text { Modal deóntico de deber }\end{array}$




$\begin{array}{ll}\text { MOD-HAB } & \text { Modal deóntico de habilidad } \\ \text { MOD-INF } & \text { Modal epistémico inferencial } \\ \text { MOD-OBL } & \text { Modal deóntico de obligación } \\ \text { NEG } & \text { Negación } \\ \text { PRO } & \text { Aspecto progresivo } \\ \text { TAN } & \text { Tiempo anterior } \\ \text { TAM } & \text { Tiempo, aspecto y modo } \\ \text { TPO } & \text { Tiempo }\end{array}$

\title{
Notas
}

1. El gullah se habla en las costas de Georgia en los Estados Unidos.

2. El sranan y el saramaca se hablan en Surinam.

3. El krio se habla en Sierra Leona, Gambia, Liberia, Ghana y Fernando Po. El wescos (también conocido como pidgin inglés de África Occidental), se habla en Nigeria, Camerún y Ghana.

4. Comrie (1976: 48-9) define la distinción entre una situación dinámica y una estativa de la siguiente manera:

\begin{abstract}
As examples to work with, we may consider first of all the verb 'know' referring a state, and the verb 'run', referring to a dynamic situation. One difference between the situations referred to these two verbs is in the relation between different phases of the situation: in the case of 'know', all phases of the situation 'John knows where I live' are identical; whichever point of the time we choose to cut in the situation. With 'run', however, this is not so: if we say 'John is running', then different phases of the situation will be very different: at one moment John will have one foot on the ground, at another moment neither foot will be on the ground and so on. Thus 'know', on the one hand, involves no change, whereas 'run' involves necessarily change.
\end{abstract}

5. La ortografía original de los ejemplos ha sido modificada de acuerdo con los grafemas propuestos por el Alfabeto Fonético Internacional (versión IPA-Kiel).

6. $\quad$ Escurre 1983: 33-56, textos 1-5.

7. $\quad$ Herzfeld 1983a: 135-7, texto 1.

8. Givón (1982: 147) señala que esta forma podría derivarse del portugués ven 'viene'.

9. En el CI de Costa Rica no ha sido registrada ninguna de estas variantes.

10. Roy (1986:151) menciona también la ocurrencia del auxiliar had como marcador verbal en el CI de Barbados. BAR: We had walk to work

(Nosotros TPO caminar a trabajo)

Habíamos ido caminando al trabajo.

11. Jones (1971), sin embargo, menciona brevemente el uso de la forma $d$ Id en krio como marcador temporal enfático.

12. En el CI de Bahamas, CI de Nicaragua, CI de Jamaica, CI de Tobago y en CI de Guyana también aparece un auxiliar preverbal d $\varepsilon$, pero este es específicamente el marcador de aspecto durativo. 
13. Aunque no se cuenta con datos suficientes para juzgar definitivamente el caso de Panamá, es probable que en esta variedad las predicaciones habituales también puedan ser expresadas por medio del marcador de progresivo. En sranan, saramaca y wescos, el progresivo es el único medio disponible para expresar predicaciones habituales.

14. Young (1973: 259) reporta que las formas $d \Lambda z$ y juistu, son los marcadores del pasado habitual en el CI de Belice. Rickford (1974: 101) también menciona el uso común de døz como marcador de habitual en esta variedad, basado en datos suministrados por un informante. Por otro lado, ni Hellinger (1979) ni Escurre (1981) mencionan el uso común de esta forma. Sin embargo, Escurre (1983:58) señala una posible "rara" ocurrencia de døz como marcador de habitual.

15. Además, en gullah y en krio, Hancock (1969 y 1987) reporta la forma blant (con sus variantes fonéticas blan, blãn y blã) como otro marcador de aspecto habitual. Propone, además, que esta forma se deriva de un uso dialectal en inglés del verbo belong to (también belang o belangt) con el sentido de be accustomed, be in the habit of (Hancock 1969: 34). Aunque, como se ha dicho, Hancock (1987) reporta el uso de este marcador en ambas variedades, Rickford (1974) menciona no encontrar trazas de este en gullah y Williams (1976) tampoco lo menciona en absoluto para el krio.

16. Modalidad es entendida como la expresión de nociones que reflejan la actitud subjetiva del hablante con respecto a lo que dice, tales como probabilidad, obligación, intención, deseo, etc.

17. El denominado futuro es considerado una categoría modal y no temporal. Cf. Comrie (1985: 43-53) para una discusión teórica acerca de las relaciones entre el tiempo futuro y el modo irrealis.

18. Esta forma también ha sido registrada para el CI de Tobago en el siglo XIX (Hancock 1987: 271).

19. Cf. Comrie 1985: 128.

\section{Bibliografía}

Agheyesi, R. 1971. West African Pidgin English. Tesis doctoral: Standford University.

Arends, J. 1986. "Genesis and development of the equative copula in Sranan”. En Muysken \& Smith (eds.): 103-27.

Bailey, B. 1966. Jamaican Creole Syntax. Cambridge: Cambridge University Press.

Bickerton, D. 1975. Dynamics of Creole System. Cambridge: Cambridge University Press.

1979. “The status of bin in the Atlantic Creoles”. En Hancock et al. (eds.): 309-14.

1980. "Creolization, linguistic universals, natural semantax and the brain". En Day (ed.): 1-18.

1981. Roots of language. Ann Arbor: Karoma.

Byrne, F. 1984. "Fi and fu: origins and fuctions in some Caribbean English-based Creoles". Lingua. 62: 97-120. 
1987. Grammatical relations in a radical creole. Amsterdam: Benjamins.

Carrington, L. et al (eds.). 1983. Studies in Caribbean Language. St. Augustine: Society for Caribbean Linguistics.

Comrie, B. 1976. Aspect. Cambridge: Cambridge University Press.

1985. Tense. Cambridge: Cambridge University Press.

Dahl, O. 1985. Tense and aspect systems. Oxford: Blackwell.

Day, R. (ed.). 1980. Issues in English Creoles: papers from the 1975 Hawaii conference. Heidelberg: Groos.

Echteld, J. 1961. The English words in Sranan. Groningen: Wolters.

Escurre, G. 1981. "Decreolization in a creole continnum: Belize”. En Highfield \& Valdmann (eds.): 27-39.

1983. Belizean Creole. En Holm (ed.): 29-70.

Gibson, K. 1986. “The ordering of auxiliary notions in Guyanese Creole”. Language 62: 571-86.

Gilbert, G. (ed.). 1987. Pidgin and Creole languages. Honolulu: University Press of Hawaii.

Givón, T. 1982. “Tense-Aspect-Modality: the creole prototype and beyond”. En Hooper (ed.): 114-63.

1984. Syntax. Amsterdam: Benjamins.

Görlach, M. \& J. Holm (eds.). 1986. Focus on the Caribbean. Amsterdam: Benjamins. Hancock, I.

1969. "A provisional comparison of the English-based Atlantic Creoles". African Language Review 8: 7-72.

1987. "A preliminary classification of the anglophone Atlantic Creoles, with syntactic data from thirty-three representative dialects". En Gilbert (ed.): 264-333.

Hancock, I. et al. (eds.). 1979. Readings in creole studies. Ghent: E. Story-Scientia.

Hellinger, M. 1979. "Across base language boundaries: the creole of Belize (British Honduras)”. En Hancock et al. (ed.): 315-33. 
Heny, F. \& B. Richards (eds.). 1983. Linguistic categories: auxiliaries and related puzzles 2. Dordrech: Reidel.

Herzfeld, A. 1978. Tense and aspect in Limon Creole: A socio-linguistic view towards a creole continuum. Tesis doctoral: University of Kansas.

1983a. “The creoles of Costa Rica and Panama”. En Holm (ed.): 131-56.

1983b. "Limon Creole and Panamian Creole: comparison and contrast". En Carrington et al. (ed.): 23-37.

Highfield, A. \& A. Valdman (eds.). 1981. Historicity and variation in Creole studies. Ann Arbor: Karoma.

Holm, J. 1978. The Creole English of Nicaragua's Miskito Coast. Tesis doctoral: University College, Nueva York.

1982. Dictionary of Bahamian English. Cold Spring: Lexis House.

(ed.). 1983. Central American English. Heidelberg: Gross.

1988/89. Pidgins and Creoles. Cambridge: Cambridge University Press.

Hooper, P. (ed.). 1982. Typological studies in language. Vol. 1: tense and aspect. Amsterdam: Benjamins.

Jones, E. 1971. “Krio: an English based language of Sierra Leone”. En Spencer (ed.): 66-94.

Jones, F. 1990. "The grammatical items bin, fo and mos in Sierra Leone Krio". Linguistics. 28: 845-66.

Mufwene, S. 1984. “Observations on time reference in Jamaican and Guyanese creoles”. English World-Wide 4: 199-229.

Muysken, P. \& N. Smith (eds.). 1986. Substrata versus universals in Creole genesis. Amsterdam: Benjamins.

Obilade, A. 1976. The nominal phrase in West African Pidgin English (Nigeria). Tesis doctoral: University of Northwestern.

O’Flynn, C. 1990. “Tiempo, aspecto y modalidad en el criollo sanandresano”. Bogotá: Universidad de los Andes.

Rickford, J. 1974. “The insights of a mesolect”. En DeCamp \& Hancock (eds.): 92-117. 
1986. "Social contact and linguistic diffusion: Hiberno English and New World Black English". Language. 62: 245-89.

Roy, J. 1986. "The structure of tense and aspect in Barbadian English Creole". En Görlach \& Holm (eds.): 141-56.

Seuren, P. 1983. “The auxiliary system in Sranan”. En Heny \& Richards (eds.): 219-51.

Shilling, A. 1975. “Bahamian English -A non-continuum?” En Day (ed.): 133-45.

Southers, D. 1975. A transformational analysis of Tobagonian Creole English. Tesis doctoral: University of North Carolina.

Spencer, J. (ed.). 1971. The English Language in West Africa. Londres: Longman.

Turner, L. 1949. Africanisms in the Gullah dialect. Ann Arbor: University of Michigan Press.

Voorhoeve, J. 1957. “The verbal system of Sranan”. Lingua. 6: 374-96.

Williams, W. 1976. Linguistics change in the syntax and semantics of Sierra Leone Krio. Tesis doctoral: Indiana University.

Young, C. 1973. A study of the creolized English spoken in the City of Belize, in its cultural and social setting. Tesis doctoral: University of York. 